\title{
SPIRAL BENDING WAVES LAUNCHED AT A VERTICAL SECULAR RESONANCE
}

\author{
WILLIAM R. WARD \\ Department of Space Studies, Southwest Research Institute, Suite 429, 1050 Walnut Street, Boulder, CO 80302 \\ AND \\ JOSEPH M. HAHN \\ Lunar and Planetary Institute, 3600 Bay Area Boulevard, Houston, TX 77058 \\ Received 2002 June 18; accepted 2003 February 19
}

\begin{abstract}
The excitation of spiral bending waves at a secular vertical resonance in a particle disk is examined. These waves are one-armed spirals of very long wavelength that are launched at sites where a secondary's nodal regression rate matches that of the disk. Nodal bending waves usually propagate radially outward as leading waves from a secular resonance exterior to the perturber, and inward as trailing waves from a secular resonance that lies interior. Their pattern speed is negative, so the spiral pattern rotates in a retrograde sense. The waves carry negative angular momentum but very little energy, and their excitation can damp the inclination of the secondary. Here we apply this theory to the case of two mutually precessing planets orbiting in a particle disk and compare their damping rate with the more familiar inclination excitation due to mean motion vertical resonances. We suggest that under certain circumstances, nodal wave damping may be an important element in maintaining planetary and/or embryo orbits in a nearly coplanar state.
\end{abstract}

Key words: celestial mechanics — planetary systems: general

\section{INTRODUCTION}

The generation of spiral waves in disk-satellite systems can cause a rapid redistribution of the angular momentum content of the disk, as well as that of any orbiting satellites. Numerous examples of this phenomenon exist in Saturn's rings, which exhibit both spiral density and spiral bending waves that are launched by orbiting satellites. The gaps in these rings suggest a history of angular momentum exchanges between ring material and satellites, and similar exchanges are expected in planet-forming disk systems. This paper continues an investigation of the variation of a secondary's inclination due to its interaction with a selfgravitating disk and examines the possible role of the $m=1$, nodal spiral bending waves as a damping mechanism. One of the main motivations of this study is to determine whether and under what circumstances the associated torques can counter the excitation of the perturber's inclination from the external vertical resonances (VRs).

This problem was examined by Borderies, Goldreich, \& Tremaine (1984, hereafter BGT84) in connection with ringsatellite inclinations. The interaction is principally at the sites of inner vertical resonances (IVRs) and outer vertical resonances (OVRs), where the vertical oscillation frequency of disk material, $\nu(r)$, equals a Doppler-shifted forcing frequency, $m \mid \Omega(r)-\Omega_{\mathrm{ps}}$, with $\Omega(r)$ being the mean motion of disk material and $\Omega_{\mathrm{ps}}$ representing the pattern speed of a particular inclination term of the Fourier-expanded disturbing potential. The disk response is to launch an $m$-armed spiral bending wave (Hunter \& Toomre 1969; Shu, Cuzzi, \& Lissauer 1983), and the reaction torques from resonances falling interior and exterior to the orbit [i.e., the external resonances at $r_{r} \approx a(1 \pm 4 / 3 m)$; see Fig. 1] were shown by BGT84 to be capable of exciting the satellite's inclination.

A similar situation had been shown to exist for a perturber's eccentricity (Goldreich \& Tremaine 1980, hereafter GT80). On the other hand, in a stellar context, Thorne (1968) concluded that cooperative effects within a thin, differentially rotating disk of stars augment the dynamical friction experienced by a massive particle (such as a molecular cloud complex), thereby damping its epicycle motions. A likely resolution was offered by Ward (1988) and Artymowicz (1993), who showed that $m>1$ Lindblad resonances fall into two broad categories regarding their effect on the secondary: the external resonances considered in GT80, and co-orbital resonances (nearly) at the secondary's orbit $a$ that damp its eccentricity, $e_{s}$. For a smooth disk surface density, $\sigma$, through the region of interest, the co-orbital resonances dominate and the eccentricity decays. A similar circumstance was then shown to prevail for VRs (see Fig. 1a), with the likely damping of the secondary's inclination (Ward \& Hahn 1994; Artymowicz 1994).

Nevertheless, the analysis of GT80 is apropos to a growing embryo or planet that eventually opens a gap in the disk by cannibalizing it, shepherding it, or both. This progressively shuts off the co-orbital resonances, and another source of damping must be sought if a low eccentricity or inclination is to be maintained. GT80 pointed out that torques associated with corotation resonances (CRs) [where the mean motion matches the pattern speed, $\left.\Omega(r)=\Omega_{\mathrm{ps}}\right]$ may still provide eccentricity damping and could slightly dominate if they do not saturate (but see also Goldreich \& Sari 2003 for a counterexample). Unfortunately, there is no analogous behavior at corotation sites in the inclination problem that could continue to oppose excitation by the external VRs. Instead, the resonant term simply adds a constant contribution to the vertical restoring force at the CR with no other effect, and BGT84 were unable to identify any other process to damp satellite inclinations. This would also appear to apply to planets or planetary embryos in a primordial planetesimal disk, and yet the nearly coplanar nature of our own planetary system is clear testimony that large inclinations were not excited by disk interactions.

The $m=1$, nodal wave is a particular type of spiral bending wave due to a VR where the disk's nodal precession rate $s(r) \equiv \Omega(r)-\nu(r)$ matches the pattern speed of the forcing 

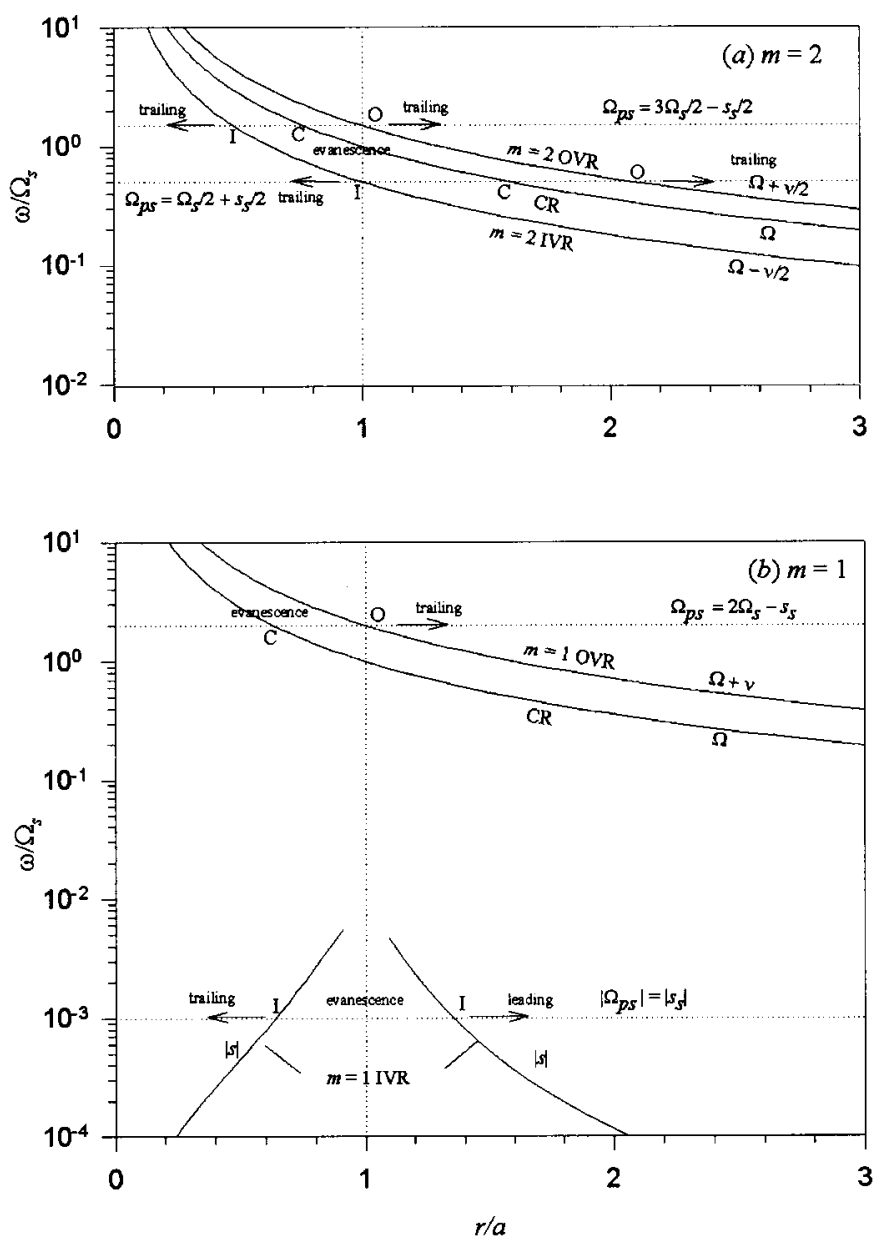

FIG. 1.-Diagram contrasting the locations of first-order vertical resonances for $m=2$ and $m=1$. The vertical axis is the resonant frequency $\omega$ normalized to the secondary's mean motion $\Omega_{s}$; the horizontal axis is orbital distance normalized to secondary's semimajor axis, $a$. The inner and outer VRs (labeled I and O) fall where $\omega=\Omega \mp \nu / m=\Omega_{\mathrm{ps}}$, in which $\nu=\Omega(r)-s(r)$ is the vertical oscillation frequency and $s(r)$ is the frequency of nodal regression. Corotation resonance (labeled C) occurs where $\omega=\Omega(r)=\Omega_{\mathrm{ps}}$. (a) The case where $m=2$. Trailing bending waves propagate away from corotation at each VR; the region of the disk between I and $\mathrm{O}$ is evanescent for that pattern speed. (b) The case where $m=1$. For the faster pattern speed there is no IVR, because $s<0$ while $\Omega_{\mathrm{ps}}>0$. However, the corresponding OVR is still in the vicinity of the planet. For the slower term, the pattern speed is much less and negative. The magnitudes of $\left|s_{s}\right|$ and $|s|$ are shown schematically, with $|s|$ increasing as the planet is approached. The IVR is where $s=s_{s}$, the site of a secular resonance. This could occur on both sides of the planet's orbit. Trailing waves are launched from an inside resonance, leading waves from an outside resonance. However, there is no CR or OVR for a negative pattern speed.

term. An example is the nodal wave train observed in Saturn's C ring generated by a mixed $e-I$ term in Titan's potential with pattern speed $\Omega_{\mathrm{ps}}=\Omega_{\mathrm{T}}-\nu_{\mathrm{T}}-\kappa_{\mathrm{T}} \approx-\Omega_{\mathrm{T}}$, where the subscript " $\mathrm{T}$ " refers to Titan and $\kappa_{\mathrm{T}}$ is its epicycle frequency (Rosen \& Lissauer 1988; Rosen 1989). Our interest here is the spiral bending wave launched at a secular resonance; strictly speaking, these resonances are of the same category as the co-orbital IVRs and also damp the inclination. However, their location is sensitive to non-Keplerian portions of the gravitational potentials and, depending on the environment, can still lie in the disk outside of a gap around a secondary (see Fig. 1b).

The theory of bending waves is reviewed in $\S 2$ and applied to an inclination secular resonance. In $\S 3$, we con- sider how to locate the secular resonance site and take as a particular case two mutually precessing objects surrounded by a particle disk. The damping of the perturbers' mutual inclination is quantified in $\S 4$, where we also compare the disk torques arising from the secular resonances with the suite of external VRs in the disk. Our findings and their implications for disk-planet systems are then summarized in $\S 5$.

\section{NODAL BENDING WAVES}

Consider a secondary mass $M_{s}$ having a semimajor axis $a$ orbiting inside a particle disk. The secondary's orbit is inclined by an angle $I_{s}$ with respect to the disk midplane, and for convenience the orbit is assumed circular. The particle disk has a surface density $\sigma(r)$ and is assumed to be semi-infinite. The secondary's gravitational potential may be expanded in the Fourier series

$$
\varphi_{s}(r, \theta, z, t)=\operatorname{Re} \sum_{l=-\infty}^{\infty} \sum_{m=0}^{\infty} \Phi_{l m}(r, z) e^{i m\left(\theta-\Omega_{\mathrm{ps}} t\right)}
$$

where $(r, \theta, z)$ are the usual cylindrical coordinates and $t$ represents time. Each Fourier component forces the disk with a pattern that rotates at angular velocity

$$
\Omega_{\mathrm{ps}} \equiv \Omega_{s}+\left(\frac{l-m}{m}\right) \nu_{s},
$$

where $\Omega_{s}$ is the secondary's angular velocity and $\nu_{s}$ is its vertical oscillation frequency (see, e.g., Shu et al. 1983).

\subsection{Wave Amplitude}

The disk can be regarded as a thin slab experiencing vertical displacements $Z(r, \theta, t)$ due to the secondary's perturbations. The equation for the disk's vertical motion is

$$
\frac{d^{2} Z}{d t^{2}}=\left(\frac{\partial}{\partial t}+\Omega \frac{\partial}{\partial \theta}\right)^{2} Z=-\frac{\partial}{\partial z}\left(\varphi_{p}+\varphi_{s}+\varphi_{d}\right)
$$

(Hunter \& Toomre 1969). The vertical acceleration of disk material due to the primary and the axisymmetric $(m=0)$ portion of the secondary's potential is represented by $-\nu^{2} Z$. The secular resonance is excited by terms in the Fourier expansion of $\partial \varphi_{s} / \partial z$ having a pattern speed independent of $\Omega_{s}$. To lowest order, only the terms with $l=0$ and $m=0$ or $m=1$ in equation (1) need be retained. The first, $\Phi_{00}$, is axisymmetric and contributes to $\nu$; the second, $\Phi_{01} \exp \left[i\left(\theta-s_{s} t\right)\right]$, is nonaxisymmetric with pattern speed $\Omega_{\mathrm{ps}}=\Omega_{s}-\nu_{s} \equiv s_{s}<0$, which is the regression frequency of the secondary's node. The restoring force due to the disk's self-gravity is $-\partial \varphi_{d} / \partial z \simeq s_{k} 2 \pi i G \sigma(\partial Z / \partial r)$, where the factor $s_{k}= \pm 1$ is chosen to satisfy boundary conditions (e.g., Shu 1984; but see also $\S 3$ below). We expect perturbations of $Z$ to take on the form $Z=\operatorname{Re}\left[H(r) \exp \left[i\left(\theta-s_{s} t\right)\right]\right]$, where the planet's ascending node is $\Omega_{\text {node }}=s_{s} t-\pi / 2$. Inserting the preceding quantities into the equation of motion 
yields the well-known wave equation

$$
-s_{k} 2 \pi i G \sigma \frac{d H}{d r}+D H=-\frac{\partial \Phi_{01}}{\partial z}
$$

applied to the $m=1$ case, for which

$$
D(r) \equiv \nu^{2}-\left(\Omega-s_{s}\right)^{2} \simeq 2 \Omega\left(s_{s}-s\right)
$$

is the distance (in units of frequency squared) from the resonance location $r_{r}$ where $D\left(r_{r}\right)=0$. The angular velocity of the disk, which differs slightly from Keplerian, is to be found from the unperturbed radial force balance,

$$
r \Omega^{2}=\frac{\partial}{\partial r}\left(\varphi_{p}+\varphi_{s}+\varphi_{d}\right) .
$$

The solution to equation (4) is a spiral wave having the form

$$
H(r)=e^{i \int k(r) d r} \int^{r} Z_{s}\left(r^{\prime}\right) e^{-i \int k\left(r^{\prime}\right) d r^{\prime}} d r^{\prime},
$$

where the integration proceeds across the disk in the direction of wave propagation and the integration limits depend on the boundary conditions adopted. The radial wavenumber and vertical forcing function are

$$
k(r)=-\frac{s_{k} D}{2 \pi G \sigma}, \quad Z_{s}(r)=-\frac{i s_{k}}{2 \pi G \sigma} \frac{\partial \Phi_{01}}{\partial z},
$$

respectively. The vertical accelerations due to the secondary are

$$
-\frac{\partial \Phi_{00}}{\partial z}=-\frac{G M_{s}}{2 a^{2}} b_{3 / 2}^{(0)} \frac{H}{a}, \quad-\frac{\partial \Phi_{01}}{\partial z}=\frac{G M_{s}}{2 a^{2}} b_{3 / 2}^{(1)} \sin I_{s}
$$

(e.g., Shu 1984), where the Laplace coefficients that appear in the potential expansion are defined by

$$
b_{j / 2}^{(m)}(\beta) \equiv \frac{2}{\pi} \int_{0}^{\infty} \frac{\cos m \varphi d \varphi}{\left(1+\beta^{2}-2 \beta \cos \varphi\right)^{j / 2}}
$$

with $\beta \equiv r / a$. The vertical forcing function is thus

$$
Z_{s}(\beta)=\frac{i s_{k}}{4} \frac{\mu_{s}}{\mu_{d}} \beta^{2} b_{3 / 2}^{(1)}(\beta) \sin I_{s},
$$

where $\mu_{s} \equiv M_{s} / M_{p}$ and $\mu_{d} \equiv \pi G \sigma / r \Omega^{2}=\pi \sigma r^{2} / M_{p}$ are the masses of the secondary and disk normalized to the mass of the primary, $M_{p}$.

Radiative boundary conditions require that $s_{k}=\operatorname{sgn}(k)$ (see Shu 1984), so equation (8) indicates that bending waves propagate in the region of the disk where $D(r) \simeq$ $2 \Omega\left(s_{s}-s\right)<0$. In many (but not all) cases, $|s|$ decreases with distance from the corotation circle at $r=a$. Thus, nodal bending waves usually propagate radially inward from a secular resonance that lies interior to corotation, and outward from an exterior secular resonance. The waves' group velocity is

$$
c_{g}=m \frac{\partial \Omega_{\mathrm{ps}}}{\partial k}=\frac{s_{k} \pi G \sigma}{m\left(s_{s}-\Omega\right)}
$$

(Toomre 1969), so an interior secular resonance generates $s_{k}=+1$ trailing waves, while an exterior secular resonance launches $s_{k}=-1$ leading waves. Recall that the pattern speed has $\Omega_{\mathrm{ps}}<0$, so the spiral pattern rotates in a retro- grade sense, whereas from equation (2) we see that all other first-order resonances $(l=m \pm 1)$ are prograde.

In Figure 1, the locations and wave properties for $m=1$ and $m=2$ are contrasted. The inner and outer VRs (designated I and O) fall where $\Omega \mp \nu / m=\Omega_{\mathrm{ps}}$; corotation resonances (designated C) occur where $\Omega(r)=\Omega_{\mathrm{ps}}$. For $m=2$ (Fig. 1a), the fast and slow pattern speeds from equation (2) are $3 \Omega_{s} / 2-s_{s} / 2$ and $\Omega_{s} / 2+s_{s} / 2$, respectively (dotted horizontal lines). For the faster pattern, VRs occur at $\left(\Omega \approx 3 \Omega_{s}\right.$, $\left.r_{\mathrm{IVR}} \approx 0.48 a\right)$ and $\left(\Omega \approx \Omega_{s}, r_{\mathrm{OVR}} \approx a\right)$; for the slower pattern, at $\left(\Omega \approx \Omega_{s}, r_{\mathrm{IVR}} \approx a\right)$ and $\left(\Omega \approx \Omega_{s} / 3, r_{\mathrm{OVR}} \approx 2.08 a\right)$. Trailing bending waves propagate away from corotation at each VR, and the region of the disk between I and O is evanescent for that pattern speed. By contrast, for $m=1$ (Fig. $1 b$ ), the fast and slow pattern speeds are $2 \Omega_{s}-s_{s}$ and $s_{s}<0$. Inner and outer VRs fall where $\Omega \mp \nu=\Omega_{\mathrm{ps}}$, or $s=\Omega_{\mathrm{ps}}$ and $\Omega-s / 2=\Omega_{\mathrm{ps}} / 2$. For the faster pattern there is no IVR, because $s<0$ while $\Omega_{\mathrm{ps}}>0$. However, the corresponding OVR is still in the vicinity of the planet, $\left(\Omega \approx \Omega_{s}, r_{\mathrm{OVR}} \approx a\right)$. For the slower term, the pattern speed is much slower and negative. In Figure $1 b$, the magnitudes of $\left|s_{s}\right|$ and $|s|$ are shown schematically, with $|s|$ increasing as the planet is approached. The IVR is where $s=s_{s}$, the site of a secular resonance. This could occur on both sides of the planet's orbit. However, there is no CR or OVR for a negative pattern speed.

Most of the contribution to the integral expression for the wave amplitude, equation (7), is due to the disk material that lies within about a wavelength of the resonance. If the waves are tightly wound, then the forcing function is nearly constant across the wave generation zone and may be pulled out of the integral. This is only marginally true for nodal waves, but we nonetheless make that assumption for convenience. Also, the wavenumber can be linearized as $k=k^{\prime} x$, where $k^{\prime}=-s_{k} r d(D / 2 \pi G \sigma) / d r \simeq s_{k}(d s / d r) / \mu_{d} \Omega$ is evaluated at resonance and $x \equiv\left(r-r_{r}\right) / r_{r}$ is the fractional distance from resonance $r_{r}$. In this approximation, the wave amplitude becomes

$$
H(\xi) \simeq-s_{k} Z_{s}\left(\frac{2 \pi r_{r}}{\left|k^{\prime}\right|}\right)^{1 / 2}\left[\frac{e^{-i \xi^{2}}}{\sqrt{\pi}} \int_{-\infty}^{\xi} e^{i \eta^{2}} d \eta\right],
$$

where $\xi \equiv-s_{k} x\left(r_{r}\left|k^{\prime}\right| / 2\right)^{1 / 2}$ is roughly the distance from resonance in wavelength units. All other quantities are to be evaluated at resonance, while the lower integration limit can usually be extended to $-\infty$ with little error. The complex quantity in brackets has a magnitude $\ll 1$ far upstream into the nonwave side of the resonance, where $\xi \ll-1$, and has a magnitude that approaches 1 downstream of the resonance, where $\xi \ll 1$. The disk's forced inclinations thus approach

$$
\begin{aligned}
\sin I_{d}=\frac{|H|}{r_{r}} & =\left|Z_{s}\right|\left|\frac{2 \pi \mu_{d} \Omega}{r d s / d r}\right|^{1 / 2} \\
& =\frac{1}{4} \frac{\mu_{s}}{\mu_{d}}\left|\frac{2 \pi \mu_{d} \Omega}{r d s / d r}\right|^{1 / 2} \beta_{r}^{2} b_{3 / 2}^{(1)} \sin I_{s}
\end{aligned}
$$

downstream of the resonance, where $\beta_{r} \equiv r_{r} / a$.

\subsection{Orbit Evolution}

The generation of bending waves requires the transfer of angular momentum between the secondary and the disk, which in turn causes the secondary's orbit to evolve. This may be assessed from the secondary's Jacobi integral 
$J=E_{s}-\Omega_{\mathrm{ps}} L_{s}$, where $E_{s}=-G M_{p} M_{s} / 2 a$ is its energy and $L_{s}=M_{s}\left(G M_{p} a\right)^{1 / 2} \cos I_{s}$ is the $z$-component of its angular momentum. Since $J$ is conserved for this system, $\dot{E}_{s}=\Omega_{\mathrm{ps}} \dot{L}_{s}$ and $\dot{a}=2 a^{2} \Omega_{\mathrm{ps}} \dot{L}_{s} / G M_{p} M_{s}$. The torque the disk exerts on the secondary is $\dot{L}_{s}$. Differentiating $L_{s}$ provides the rate at which the secondary's inclination varies:

$$
\sin I_{s} \frac{d I_{s}}{d t}=-\frac{\dot{L}_{s}}{M_{s} a^{2} \Omega_{s}}\left(1-\frac{\Omega_{\mathrm{ps}}}{\Omega_{s}} \cos I_{s}\right)
$$

(cf. BGT84). Note that because $\Omega_{\mathrm{ps}}=s_{s}<0$ where $\left|s_{s}\right| \ll \Omega_{s}$, $\Delta a / a \sim\left(-s_{s} / \Omega_{s}\right) \Delta I_{s}^{2}$, that is, the semimajor axis is relatively unaffected by the nodal wave. This is not unexpected, given that we are dealing with a secular resonance, and is another distinguishing characteristic when compared with $(m>1)$ VRs with faster pattern speeds.

The density of angular momentum transported by spiral bending waves is $j=m^{2}\left(\Omega_{\mathrm{ps}}-\Omega\right) \sigma|H|^{2} / 2$ (Bertin \& Mark 1980), so the total flux of angular momentum that waves deliver across a ring of radius $r$ is

$$
F=2 \pi r c_{g} j=s_{k} m G r(\pi \sigma H)^{2}=2 \pi m s_{k} \frac{\mu_{d}^{3} Z_{s}^{2}(r \Omega)_{r}^{5}}{G|r d s / d r|} .
$$

Since the secondary is the source of this angular momentum flux, $\dot{L}_{s}=-\operatorname{sgn}(j)|F|$; for $m=1, \Omega_{\mathrm{ps}}=s_{s} \ll \Omega, j$ is negative, $\dot{L}_{s}$ is positive, and the inclination decays. Inserting these quantities into equation (15) yields the rate at which this resonance damps the secondary's inclination,

$$
\begin{aligned}
\frac{1}{\sin I_{s}} \frac{d I_{s}}{d t} & =-2 \pi \frac{\mu_{s}}{\mu_{d}}\left(\mu_{d} Z_{s}\right)^{2} \beta^{-1}\left|\frac{\Omega}{r d s / d r}\right| \Omega_{s} \\
& =-\frac{\pi}{8} \mu_{s} \mu_{d} \beta_{r}^{3}\left(b_{3 / 2}^{(1)}\right)^{2}\left|\frac{\Omega}{r d s / d r}\right| \Omega_{s} .
\end{aligned}
$$

We note in passing that Ward \& Hahn (1998) investigated the closely related phenomenon of spiral density waves launched at an apsidal secular resonance. This resonance lies where the precession, $g=d \tilde{\omega} / d t$, of the disk's longitude of periapse $\tilde{\omega}$ matches the corresponding precession rate of the secondary, $g_{s}$, and horizontal waves are excited in the disk similarly to equation (14) but instead proportional to the secondary's eccentricity. The generation of these waves will damp the secondary's eccentricity, $e_{s}$, at the rate ${ }^{1}$

$$
\frac{1}{e_{s}} \frac{d e_{s}}{d t}=-\frac{\pi}{8} \mu_{s} \mu_{d} \beta_{r}^{3}\left(b_{3 / 2}^{(2)}\right)^{2}\left|\frac{\Omega}{r d g / d r}\right| \Omega_{s},
$$

which revises the rate given in Ward \& Hahn (1998) to account for an omitted factor of $\frac{1}{2}$. This rate was also found by Tremaine (1998).

\subsection{Fate of the Waves}

An important assumption implicit in equation (17) is that the waves propagate out of the resonance zone without return. We first check to see if $|k|^{-1}$ remains larger than the disk scale height, $h \approx v_{\text {disp }} / \Omega$, where $v_{\text {disp }}$ is the particles' dispersion velocity; this is a necessary condition for the propagation of long waves. From the dispersion relationship, $|k| \sim\left(\left|s_{s}\right| \Omega / \pi G \sigma\right)\left(1-\left|s / s_{s}\right|\right) \equiv k_{*}\left(1-\left|s / s_{s}\right|\right)$. The wavenum-

\footnotetext{
${ }^{1}$ We wish to correct footnote 9 in that work. As in the case described here, both exterior and interior resonances generate waves of negative angular momentum density and thus damp $e_{s}$.
}

ber initially rises as $|s|$ drops, but it then changes more slowly as $k_{*} \sim r^{-1}\left(\left|s_{s}\right| / \mu_{d} \Omega\right) \propto 1 / \sigma r^{3 / 2}$. Accordingly, the product $k_{*} h \sim v_{\text {disp }}\left|s_{s}\right| / \pi G \sigma$. For a particle disk in collisional equilibrium, $v_{\text {disp }}$ is comparable to the escape velocity, $v_{\text {esc }} \sim(8 \pi G \rho / 3)^{1 / 2} R \approx 10^{2}[R /(1 \mathrm{~km})] \mathrm{cm} \mathrm{s}^{-1}$, from individual particles of characteristic radius $R$. Thus, the product $k_{*} h \approx 3 \times 10^{-5}[R /(1 \mathrm{~km})][r /(1 \quad \mathrm{AU})]^{1 / 2}\left(\left|s_{s}\right| / \mu_{d} \Omega\right) \propto R / \sigma$. For cases of interest such that $\left|s_{s}\right| / \Omega_{r}$ and $\mu_{d}\left(r_{r}\right)$ are not too different, inward-traveling waves are supported by the disk, whereas outward-traveling waves may eventually violate the criterion as $\sigma$ decreases sufficiently with distance. On the other hand, solid-body accretion becomes slower with distance, making it likely that the characteristic particle size $R$ will decrease as well. There is a minimum dispersion velocity of $v_{\text {crit }} \approx \pi G \sigma / \Omega$ required for gravitational stability of the particle layer. If $v_{\text {esc }}$ drops below this, then collective gravity sets the velocity dispersion and $k_{*} h \rightarrow\left|s_{s}\right| / \Omega \propto r^{3 / 2}$, which is independent of $\sigma$ and approaches unity only at very large distance, $r_{Q} / r_{r} \approx\left(\Omega_{r} /\left|s_{S}\right|\right)^{2 / 3}$. We restrict our attention to disks that do not extend that far.

If there is a physical edge to the disk, $r_{d}<r_{Q}$, that can reflect nodal waves while they are still long waves (i.e., $k \rightarrow-k, c_{q} \rightarrow-c_{q}$ ), they will return to the resonance, where their angular momentum flux is reabsorbed by the planet (Tanaka, Takeuchi, \& Ward 2000; Goldreich \& Sari 2003; Ward 2003). In the absence of dissipation, the net torque shuts off and the inclination no longer decays. ${ }^{2}$ This requires a fairly sharp drop in density, that is, $|d \ln \sigma / d \ln r| \gg r|k| \propto$ $1 / \sigma r^{1 / 2}$, but may well be the fate of inward-propagating waves if they encounter interior regions already depleted by other forming planets. On the other hand, outwardtraveling waves can travel much farther, and if these waves dissipate over the round-trip, equation (17) remains valid. In a disk of low optical depth, the most likely source of dissipation is nonlinear effects; that is, waves will break when $|k|^{-1}$ becomes comparable to the wave amplitude $H$ (e.g., Shu 1984). From the wave-amplitude equation (eq. [14]) and the conservation-of-action equation (eq. [16]), we can write $|H|=(|F| / G r)^{1 / 2} / \pi \sigma \sim r_{r} \sin I_{d}\left(\sigma_{r} / \sigma\right)\left(r_{r} / r\right)^{1 / 2}$, so that the product $|k H| \rightarrow \sin I_{d}\left[\left|s_{s}\right| / \mu_{d}\left(r_{r}\right) \Omega_{r}\right]\left(\sigma_{r} r_{r} / \sigma r\right)^{2}$. At some point, the disk must have a region where the surface density ramps down faster than $r^{-2}$ to avoid mass divergence. However, the more remote the region, the larger the density gradient necessary for efficient reflection. If this is not attained, the waves penetrate the region with $|k H| \propto(\sigma r)^{-2}$ increasing faster than $r^{2}$, so that the waves eventually become nonlinear and dissipate; it is this case that is considered in the remainder of the paper.

\section{SECULAR RESONANCE SITES}

Further progress requires locating the resonance, which is the site where the disk's nodal rate matches the planet's rate: $s(r)=s_{s}$. Unlike mean motion resonances, the location of a secular resonance is sensitive to non-Keplerian aspects of the potentials. Thus, careful treatments of the precession rates of both secondary and disk are required.

${ }^{2}$ A reduced torque occurs for partial dissipation, in which case eq. (17) would be replaced by an expression that depended on this aspect as well. We treat the interesting case of long-wave reflection at a physical edge in another work (Ward 2003). 


\subsection{Precession of the Disk, $s(r)$}

The potential of the perturber affects both the vertical $\left[\nu^{2} \equiv \partial^{2}\left(\varphi_{p}+\varphi_{s}\right) /\left.\partial z^{2}\right|_{z=0}\right]$ and the orbital $\left[\Omega^{2} \equiv r^{-1} \partial\left(\varphi_{p}+\right.\right.$ $\left.\left.\varphi_{s}\right) / \partial r\right]$ frequencies of the disk:

$$
\begin{gathered}
\nu^{2}=\Omega_{\mathrm{K}}^{2}+\frac{1}{H} \frac{\partial \Phi_{00}}{\partial z}=\Omega_{K}^{2}+\frac{G M_{s}}{2 a^{3}} b_{3 / 2}^{(0)}, \\
\Omega^{2}=\Omega_{\mathrm{K}}^{2}+\frac{1}{r} \frac{\partial \Phi_{00}}{\partial r}=\Omega_{\mathrm{K}}^{2}+\frac{G M_{s}}{2 a^{3}}\left(b_{3 / 2}^{(0)}-\beta^{-1} b_{3 / 2}^{(1)}\right),
\end{gathered}
$$

where $\Omega_{\mathrm{K}} \equiv\left(G M_{p} / r^{3}\right)^{1 / 2}$ is the usual Keplerian frequency, with $M_{p}$ being the primary's mass. Assuming $s \ll \Omega$, $s=\left(\Omega^{2}-\nu^{2}\right) /(\Omega+\nu) \approx\left(\Omega^{2}-\nu^{2}\right) / 2 \Omega$. The secondary increases the vertical frequency slightly more than it does the orbital frequency, and consequently the nodes regress at the well-known rate

$$
s \simeq-\frac{1}{4} \mu_{s} \beta^{2} b_{3 / 2}^{(1)} \Omega .
$$

What about the mass of the disk itself? Clearly, its selfgravity can also affect both its vertical and azimuthal frequencies. Replacing the mass of the secondary with the mass of a disk annulus, $M_{s} \rightarrow 2 \pi \sigma r^{\prime} d r^{\prime}$ and $a \rightarrow r^{\prime}$, the form of equation (20) implies an additional contribution of

$$
\pi G \int_{\text {disk }} \frac{\sigma\left(r^{\prime}\right)}{r^{\prime 2}}\left(b_{3 / 2}^{\prime(0)}-\beta^{\prime-1} b_{3 / 2}^{\prime(1)}\right) d r^{\prime}
$$

to $\Omega^{2}$, where now $b_{3 / 2}^{\prime(m)} \equiv b_{3 / 2}^{(m)}\left(r / r^{\prime}\right)$ and $\beta^{\prime} \equiv r / r^{\prime}$. The vertical acceleration from the disk's self-gravity is

$$
\begin{aligned}
\frac{-\partial \varphi_{d}}{\partial z} & =-G \int \sigma\left(r^{\prime}\right) r^{\prime} d r^{\prime} \int_{-\pi}^{\pi} d \theta \frac{H\left(r^{\prime}\right) \cos \theta-H(r)}{\left(r^{\prime 2}+r^{2}-2 r r^{\prime} \cos \theta\right)^{3 / 2}} \\
& =-\pi G \int \frac{\sigma\left(r^{\prime}\right)}{r^{\prime 2}} d r^{\prime}\left[b_{3 / 2}^{(1)} H\left(r^{\prime}\right)-b_{3 / 2}^{(0)} H(r)\right]
\end{aligned}
$$

(e.g., Shu 1984). Rewriting the bracketed quantity as $b_{3 / 2}^{(1)}\left[H\left(r^{\prime}\right)-\beta^{\prime-1} H(r)\right]+\left(\beta^{\prime-1} b_{3 / 2}^{(1)}-b_{3 / 2}^{(0)}\right) H(r)$, the second term leads to the same contribution to $\nu^{2}$ as equation (22) and therefore does not alter $\Omega^{2}-\nu^{2}$. In the first integral, however, $b_{3 / 2}^{(1)}$ is very strongly peaked at $\left(\beta^{\prime}=1, r^{\prime}=r\right)$. This is the term that leads to the first derivative in equation (4). At very small $x=\left(r^{\prime}-r\right) / r$, any thickness $h=h^{\prime} r$ to the disk cannot be ignored. We soften the Laplace coefficients by adding $h^{2}$ to the denominator of equation (10) and then replace it with its asymptotic form, $\quad b_{3 / 2}^{(m)} \rightarrow 2 r^{\prime 2} /$ $\pi\left[\left(r^{\prime}-r\right)^{2}+h^{2}\right]=2 / \pi\left(x^{2}+h^{\prime 2}\right)$. Setting $r^{\prime}=r$ elsewhere, extending the integration to $(-\infty, \infty)$, and pulling slowly varying terms out of the integral yields

$$
\begin{aligned}
2 G \sigma \int_{-\infty}^{\infty} \frac{H(r)-H\left(r^{\prime}\right)}{\left(r^{\prime}-r\right)^{2}+h^{2}} d r^{\prime} \\
\quad=\frac{2 \pi G \sigma}{h} H(r)-2 G \sigma \int_{-\infty}^{\infty} \frac{H\left(r^{\prime}\right)}{\left(r^{\prime}-r\right)^{2}+h^{2}} d r^{\prime} .
\end{aligned}
$$

The first term acting alone would contribute an additional term of order $-\pi G \sigma / h \Omega$ to the disk's precession rate. This would pertain if adjacent parts of the disk were either unperturbed, $H\left(r^{\prime}\right)=0$, or uncorrelated (e.g., Ward 1981). However, because nearby disk material executes very similar motion in the coherent wave, the second integral is of comparable importance (A. Toomre 2002, private communication). ${ }^{3}$ It can be evaluated through residue theory ${ }^{4}$ as $-2 \pi G \sigma H(r+i h) / h$. If $h \ll r$, we can replace $H(r+i h)$ with its Taylor expansion, so that to first-order accuracy,

$$
-2 G \sigma \int_{-\infty}^{\infty} \frac{H\left(r^{\prime}\right)-H(r)}{\left(r^{\prime}-r\right)^{2}+h^{2}} d r^{\prime}=-2 \pi i G \sigma \frac{d H}{d r} .
$$

The result becomes exact in the limit $h \rightarrow 0$ and simply recovers the first term in the wave equation (eq. [4]). We conclude that, aside from this term, the self-gravity of the disk is negligible compared with the secondary in its effect on its own precession rate, $s(r)$.

\subsection{Precession of the Secondary, $s_{s}$}

In order to ascertain the effect of the disk on the precession of the planet, we will employ a direct approach of deriving the reaction forces on the planet by integrating over the perturbed disk. The gravitational potential due to the interaction of the secondary $M_{s}$ with a disk mass element $\delta m=\sigma r d r d \theta$ is

$$
\delta \varphi=-G \delta m /\left|\boldsymbol{r}-\boldsymbol{r}_{s}\right|,
$$

where $\quad \boldsymbol{r}=r \hat{\boldsymbol{n}}+z \hat{\boldsymbol{k}}, \quad \boldsymbol{r}_{s}=a \hat{\boldsymbol{n}}+z_{s} \hat{\boldsymbol{k}}, \quad\left|\boldsymbol{r}-\boldsymbol{r}_{s}\right| \simeq\left[r^{2}+a^{2}-\right.$ 2 ar $\left.\cos \left(\theta-\theta_{s}\right)+\left(z-z_{s}\right)^{2}\right]^{1 / 2}$, and the indirect term has been omitted because it contains no secular contribution (e.g., Brouwer \& Clemence 1961). The resulting forces on the planet are

$$
\begin{aligned}
&\left\{\delta F_{r}, \delta F_{\theta}, \delta F_{z}\right\}=\frac{G M_{s} \sigma r d r d \theta}{\left|\boldsymbol{r}-\boldsymbol{r}_{s}\right|^{3}}\left\{r \cos \left(\theta-\theta_{s}\right)-a,\right. \\
&\left.r \sin \left(\theta-\theta_{s}\right), z-z_{s}\right\} .
\end{aligned}
$$

Assuming $z$ and $z_{s}$ are small, they are ignored in the denominator, which can then be Fourier-expanded as

$$
\begin{aligned}
& {\left[r^{2}+a^{2}-2 \operatorname{arcos}\left(\theta-\theta_{s}\right)\right]^{-3 / 2}} \\
& =\frac{1}{a^{3}}\left[\frac{b_{3 / 2}^{(0)}}{2}+\sum_{m=1}^{\infty} b_{3 / 2}^{(m)} \cos m\left(\theta-\theta_{s}\right)\right] .
\end{aligned}
$$

Integrations of $\delta F_{r}$ and $\delta F_{\theta}$ over $\theta$ (i.e., $d F_{i} \equiv \int_{0}^{2 \pi} \delta F_{i} d \theta$ ) are easily done to find the forces due to an annulus of the disk,

$$
d F_{r}=\frac{\pi G M_{s}}{a^{3}} r \sigma d r\left(r b_{3 / 2}^{(1)}-a b_{3 / 2}^{(0)}\right), \quad d F_{\theta}=0 .
$$

Consequently, there is no dependence of these forces on the angular position of the planet, $\theta_{s}$, to lowest order in $z$. The

\footnotetext{
${ }^{3}$ A similar result was found by Ward \& Hahn (1998) for apsidal waves, wherein the effect of the perturbed disk potential on precession of a test particle almost cancels that of the unperturbed disk potential.

${ }^{4}$ Analytically extend $H(z)$ to the complex plane and choose a semicircular integration path with its base on the real axis and the return path on the $+i$ side. The denominator can be factored as $1 /$ $(z-r+i h)(z-r-i h)$ with two singularities, but only one $(z=r+i h)$ is inside the contour. Thus, Cauchy's integral theorem, $\oint f(z) /(z-a) d z=$ $2 \pi i f(a)$, leads to the desired result.
} 
vertical force

$$
\begin{aligned}
d F_{z}= & \frac{G M_{s} \sigma r d r}{a^{3}} \\
& \times \int_{0}^{2 \pi}\left(z-z_{s}\right)\left[\frac{b_{3 / 2}^{(0)}}{2}+\sum_{m=1}^{\infty} b_{3 / 2}^{(m)} \cos m\left(\theta-\theta_{s}\right)\right] d \theta
\end{aligned}
$$

is more subtle, however, owing to the dependence of $z$ on $\theta-\theta_{s}$.

The vertical motion of the planet is $z_{s}=\operatorname{Re}\left(a \sin I_{s}\right.$ $\left.\exp \left[i\left(\theta_{s}-s_{s} t\right)\right]\right) . \quad$ Substituting $z=\operatorname{Re}\left(H \exp \left[i\left(\theta-s_{s} t\right)\right]\right)$ into equation (30) and writing $\exp \left[i\left(\theta-s_{s} t\right)\right]=$ $\exp \left[i\left(\theta_{s}-s_{s} t\right)\right] \exp \left[i\left(\theta-\theta_{s}\right)\right]$ yields the azimuthally integrated results,

$$
\begin{aligned}
d F_{z} & =\operatorname{Re}\left[\frac{\pi G M_{s} \sigma r d r}{a^{3}}\left(H b_{3 / 2}^{(1)}-a \sin I_{s} b_{3 / 2}^{(0)}\right) e^{i\left(\theta_{s}-s_{s} t\right)}\right] \\
& \equiv d F_{H}-d F_{s}
\end{aligned}
$$

where $H$ is given by equation (13). The quantity $d F_{S}$ can be written simply,

$$
d F_{s}=\frac{\pi G M_{s} \sigma r d r}{a^{2}} b_{3 / 2}^{(0)} \sin I_{s} \cos \left(\theta_{s}-s_{s} t\right) .
$$

To determine the effect on the orbit, we can use Gauss's form of Lagrange's equations:

$$
d \dot{I}=-\frac{d W}{a \Omega_{s}} \sin \left(\theta_{s}-s_{s} t\right), \quad d s_{s}=\frac{d W}{a \Omega_{s} \sin I_{s}} \cos \left(\theta_{s}-s_{s} t\right)
$$

(e.g., Brouwer \& Clemence 1961), where

$$
\begin{aligned}
d W & =-\left(d F_{r} / M_{S}\right) \sin I_{s} \cos \left(\theta_{s}-s_{s} t\right)+\left(d F_{z} / M_{s}\right) \cos I_{s} \\
& \approx-\frac{\pi G r \sigma d r}{a^{3}} r b_{3 / 2}^{(1)} \sin I_{s} \cos \left(\theta_{s}-s_{s} t\right)+\frac{d F_{H}}{M_{s}}
\end{aligned}
$$

is the acceleration perpendicular to the orbit plane. One can see that the first term in $d W$ will not contribute to $\langle d \dot{I}\rangle$ when averaged over the planet's orbit, but it does contribute an amount

$$
\left\langle d s_{S}\right\rangle=-\frac{\pi G r \sigma d r}{2 a^{4} \Omega_{s}} r b_{3 / 2}^{(1)}
$$

to the precession. Since the Laplace coefficient becomes very large as the planet is approached, as $O\left(2 a^{2} /\right.$ $\left.\pi\left[(r-a)^{2}+h^{2}\right]\right)$, where $h$ is the disk thickness, equation (35) alone predicts a precession rate of order $\pi G \sigma / 2 h \Omega_{s}$.

Next consider $F_{H}$; the contributions it makes to $\dot{I}_{s}$ and $s_{s}$ are respectively

$$
\begin{aligned}
& \left\langle d \dot{I}_{s}\right\rangle=\frac{\pi G \sigma r d r}{2 a^{4} \Omega_{s}} b_{3 / 2}^{(1)} \operatorname{Im}(H), \\
& \left\langle d s_{s}\right\rangle=\frac{\pi G \sigma r d r}{2 a^{4} \Omega_{s} \sin I_{s}} b_{3 / 2}^{(1)} \operatorname{Re}(H) .
\end{aligned}
$$

The imaginary part of $H$ occurs mostly near resonance, as the nodes of the disk material rotate with the waveform. Integrating equation (13) radially over the disk, it becomes

$$
\begin{aligned}
\int_{\mathrm{disk}} H(r) d r \approx & -s_{k} Z_{S}\left(\frac{2 \pi r}{\left|k^{\prime}\right|}\right)^{1 / 2} r\left(\frac{2}{r k^{\prime}}\right)^{1 / 2} \\
& \times \int_{-\infty}^{\infty} d \xi\left(\frac{e^{-i \xi^{2}}}{\sqrt{\pi}} \int_{-\infty}^{\xi} e^{i \eta^{2}} d \eta\right) .
\end{aligned}
$$

To evaluate the double integral, we make the coordinate transformations $u=(\xi+\eta) / \sqrt{2}$ and $v=(\xi-\eta) / \sqrt{2}$, yielding

$$
\int_{-\infty}^{0} d v \int_{-\infty}^{\infty} e^{-2 i u v} d u=\int_{-\infty}^{0} \pi \delta(v) d v=\frac{\pi}{2}
$$

where $\delta(v)$ denotes the Dirac delta function. The factor of $\frac{1}{2}$ comes from the fact that we only have half of $\delta(v)$ in the range of integration. Combining equations (37) and (38) with equations (11) and (36) and the definition $\left|r k^{\prime}\right|=|r d s / d r| / \mu_{d} \Omega$ then recovers equation (17) for $d I_{s} / d t$.

The purely imaginary value (due to $Z_{s}$ ) for equation (38) seems to imply no contribution to $s_{s}$, leaving equation (35) unopposed. But this is due to our neglect of any variation in $Z_{s}$ and its removal from the integral. Again, the rationale for this was that most of the imaginary contribution occurs near the resonance. This is not so for the real part; ignoring the lead selfgravitating term in equation (4) for $r \ll r_{r}$ yields

$$
\begin{aligned}
H(r) \approx \frac{i Z_{s}}{k} & =-\frac{\partial \Phi_{01} / \partial z}{D} \\
& =\frac{\mu_{s} \beta^{2} r b_{3 / 2}^{(1)} \Omega}{4\left(s_{s}-s\right)} \sin I_{s}=-r\left(\frac{s}{s_{s}-s}\right) \sin I_{s},
\end{aligned}
$$

where equation (21) is employed in the last step. This is the so-called nonwave part of the solution. Substituting into equation (36) and combining with equation (35) yields

$$
\left\langle d s_{s}\right\rangle=-\frac{\pi G \sigma r d r}{2 a^{4} \Omega_{s}} r b_{3 / 2}^{(1)}\left(\frac{s_{s}}{s_{s}-s}\right) .
$$

This expression no longer diverges as the planet is approached, that is, $\left\langle d s_{s}\right\rangle \rightarrow-2 s_{s}\left(\mu_{d} / \mu_{s}\right) d r / a$. Note that this is positive, so that the near parts of the disk try to advance the planet's node. This is because the nonwave solution describes disk annuli that are forced to align with the planet; on the far side of the resonance, the situation is reversed. Equation (40) has a singularity at $s=s_{s}$, an unphysical result because this is the region where the self-gravity organizes the motion and prevents divergence. Nevertheless, we can use this expression to crudely estimate the contribution to $s_{s}$ by integrating over the disk and taking the principal value of the integral:

$$
\Delta s_{s}=\frac{-\pi G}{2 a^{4} \Omega_{s}} \mathrm{PV} \int_{a}^{\infty} \sigma r^{2} b_{3 / 2}^{(1)}\left(\frac{s_{s}}{s_{s}-s}\right) d r
$$

Using equation (21), again adopting the asymptotic form for $b_{3 / 2}^{(1)} \approx 2 / \pi\left(x^{2}+h^{\prime 2}\right)$, and evaluating all other 
quantities at $r=a$, equation (41) becomes

$$
\begin{aligned}
\Delta s_{s} & =\frac{-\pi G \sigma}{2 a \Omega_{s}} \mathrm{PV} \int_{a}^{\infty} \frac{b_{3 / 2}^{(1)} d r}{1-s / s_{s}} \\
& =-\frac{G \sigma}{a \Omega_{s}} \mathrm{PV} \int_{0}^{\infty} \frac{d x}{x^{2}+h^{\prime 2}-\mu_{s} \Omega_{s} / 2 \pi\left|s_{s}\right|} .
\end{aligned}
$$

The normalized disk thickness $h^{\prime} \equiv h / r \sim v_{\text {disp }} / r \Omega$, where $v_{\text {disp }}$ represents the particles' dispersion velocity. Assuming, for instance, that this is on the order of the particles' escape velocity gives $h^{\prime} \approx 10^{-3}[R /(10 \mathrm{~km})][\mathrm{r} /$ $(10 \mathrm{AU})]^{1 / 2}\left[M_{p} /\left(1 M_{\odot}\right)\right]^{-1 / 2}$ for a particle radius $R$. If $\left|s_{s}\right|>\mu_{s} \Omega_{s} / 2 \pi h^{\prime 2} \equiv s_{\text {crit }}$, there is no pole in the denominator, and

$$
\Delta s_{s}=\frac{-\pi G \sigma}{2 h \Omega} \frac{1}{\sqrt{1-s_{\text {crit }} /\left|s_{S}\right|}} .
$$

The disk itself can provide the necessary precession for equation (43) to apply only if $\pi G \sigma / h \Omega \gg s_{\text {crit }}$, which implies a massive disk compared with the secondary, that is, $\pi \sigma r^{2} / M_{s} \gg 1.6 \times 10^{2}[R /(10 \mathrm{~km})]^{-1}[r /(10 \mathrm{AU})]^{-1 / 2}$ $\left[M_{p} /\left(1 M_{\odot}\right)\right]^{1 / 2}$. However, if $\left|s_{s}\right|<s_{\text {crit }}$, a resonance exists and the principal value of the integral vanishes. A more accurate evaluation of equation (42) yields a small nonzero value, but the overall effect of the disk is small.

\subsection{Two-Planet Problem}

Thus, for small disks the precession of a secondary depends mostly on other aspects of its specific environment. We take as an important application two mutually precessing planets, $M_{1}$ and $M_{2}$, with relative inclination $I_{s}$. In isolation, they precess at the rate

$$
s_{s}=-\frac{1}{4} \mu_{1} \alpha b_{3 / 2}^{(1)}(\alpha) \Omega_{2}-\frac{1}{4} \mu_{2} \alpha^{2} b_{3 / 2}^{(1)}(\alpha) \Omega_{1}
$$

about their combined angular momentum vector with antialigned nodes (e.g., Brouwer \& Clemence 1964), where $a_{1}<a_{2}$ are the semimajor axes, $\mu_{i}=M_{i} / M_{p}$, the $\Omega_{i}$ are their mean motions, and $\alpha \equiv a_{1} / a_{2}$. Their individual inclinations to the invariable plane must satisfy $M_{1} a_{1}^{2} \Omega_{1} \sin I_{1}=$ $M_{2} a_{2}^{2} \Omega_{2} \sin I_{2}$, so that for small inclinations

$$
\sin I_{i} \approx \frac{\mu_{j} \sqrt{a_{j}} \sin I_{s}}{\mu_{1} \sqrt{a_{1}}+\mu_{2} \sqrt{a_{2}}}
$$

where $i \neq j$. Now add a disk whose unperturbed orientation is in the invariable plane. Because there are two perturbers, equations (11) and (22) must be generalized to include both, that is,

$$
\begin{gathered}
s \simeq-\frac{1}{4}\left[\mu_{1} \beta_{1}^{2} b_{3 / 2}^{(1)}\left(\beta_{1}\right)+\mu_{2} \beta_{2}^{2} b_{3 / 2}^{(1)}\left(\beta_{2}\right)\right] \Omega \\
Z_{s}=\frac{i s_{k}}{4} \frac{\mu_{1} \mu_{2}}{\mu_{d}}\left[\frac{\sqrt{a_{1}} \beta_{2}^{2} b_{3 / 2}^{(1)}\left(\beta_{2}\right)-\sqrt{a_{2}} \beta_{1}^{2} b_{3 / 2}^{(1)}\left(\beta_{1}\right)}{\mu_{1} \sqrt{a_{1}}+\mu_{2} \sqrt{a_{2}}}\right] \sin I_{s}
\end{gathered}
$$

where $\beta_{i} \equiv r / a_{i}$. These quantities can also be written in terms of $\alpha_{i} \equiv 1 / \beta_{i}=a_{i} / r$ by noting from their definition that $\beta^{3 / 2} b_{3 / 2}^{(1)}(\beta)=\alpha^{3 / 2} b_{3 / 2}^{(1)}(\alpha)$. Well inside $a_{1}$, the Laplace coefficients can be approximated as $b_{3 / 2}^{(1)}\left(\beta_{i}\right) \approx 3 \beta_{i}$ and $s \rightarrow-\frac{3}{4} r^{3} \Omega\left(\mu_{1} / a_{1}^{3}+\mu_{2} / a_{2}^{3}\right) \propto r^{3 / 2}$, while far outside $a_{2}$, $b_{3 / 2}^{(1)}\left(\alpha_{i}\right) \approx 3 \alpha_{i}$ and $s \rightarrow-\frac{3}{4} r^{-2} \Omega\left(\mu_{1} a_{1}^{2}+\mu_{2} a_{2}^{2}\right) \propto r^{-7 / 2}$. In

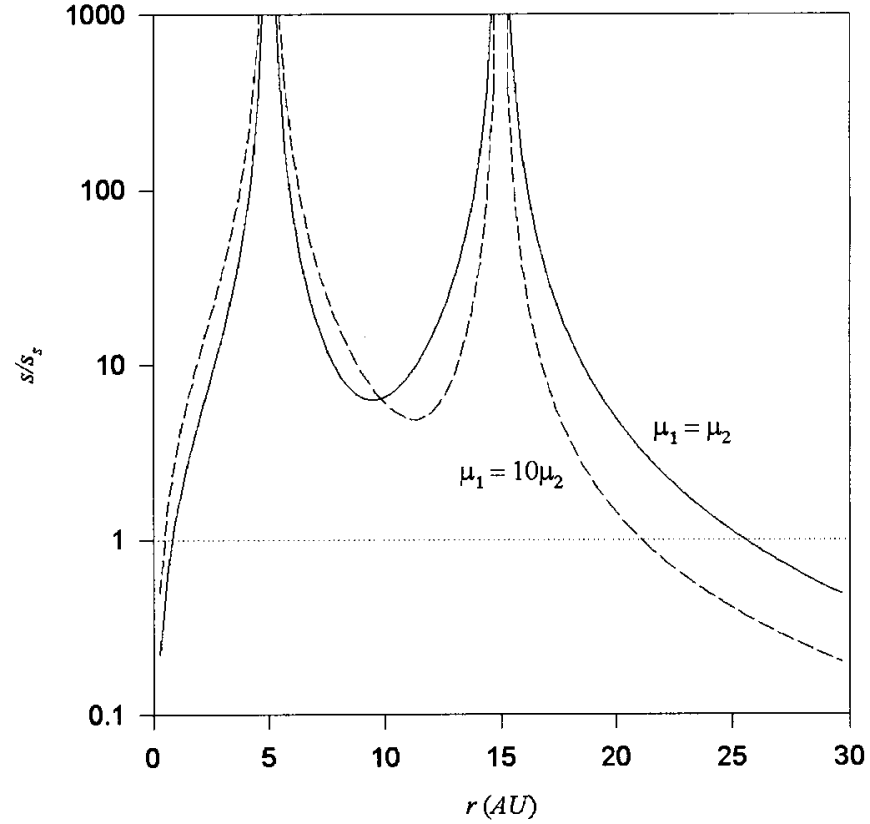

FIG. 2.-Relative precession rate $s / s_{s}$ for the two examples of the twoplanet problem described in $\S 4$. The mutual precession of the planets, $s_{s}$, is given by eq. (44); the precession of disk particles, $s$, is found from eq. (46) and diverges as each planet is approached at 5 and 15 AU. Secular resonances occur where the curves cross unity, i.e., $s / s_{s}=1$; there is one interior and one exterior to the pair.

Figure 2, the ratio

$$
\frac{s}{s_{s}}=\frac{\beta_{1}^{1 / 2}\left[b_{3 / 2}^{(1)}\left(\beta_{1}\right)+\hat{\mu} \alpha^{2} b_{3 / 2}^{(1)}\left(\alpha \beta_{1}\right)\right]}{\alpha^{2}\left(\alpha^{1 / 2}+\hat{\mu}\right) b_{3 / 2}^{(1)}(\alpha)}
$$

is plotted for two example problems described in $\S 4$, where $\hat{\mu}=\mu_{2} / \mu_{1}$. At each planet there are local maxima of order $|s| \approx \mu_{i} \Omega / 2 \pi h^{2} \gg\left|s_{s}\right|$, so that the curves are strongly peaked at these positions. Resonance occurs when the curve passes through unity; thus, there should be a resonance both interior and exterior to the pair. ${ }^{5}$

Concentrating on the external resonance for specificity, and adopting a situation in which Laplace coefficients of $\alpha$ can be approximated by a power of $r$ as above (i.e., $\alpha \ll 1$ ), so that

$$
\begin{gathered}
s_{s} \approx-\frac{3}{4} \Omega_{2} \alpha^{3 / 2}\left(\mu_{1} \alpha^{1 / 2}+\mu_{2}\right), \\
s \approx-\left[\frac{3}{4} \mu_{1} \alpha^{2}\left(a_{2} / r\right)^{2}+\frac{1}{4} \mu_{2}\left(a_{2} / r\right) b_{3 / 2}^{(1)}\left(\alpha_{2}\right)\right] \Omega,
\end{gathered}
$$

the resonance position is found from

$$
\mu_{1} \alpha^{2}\left[1-\left(\frac{a_{2}}{r}\right)^{7 / 2}\right]=\mu_{2}\left[\frac{1}{3}\left(\frac{a_{2}}{r}\right)^{5 / 2} b_{3 / 2}^{(1)}\left(\alpha_{2}\right)-\alpha^{3 / 2}\right] .
$$

\section{DISCUSSION \\ 4.1. Applications}

Consider first the case where $\mu_{1} \alpha^{1 / 2} \gg \mu_{2}$, for which equation (46) nearly reduces to equation (11) and the $\alpha^{3 / 2}$

\footnotetext{
${ }^{5}$ One can show, however, that there is no resonance between the two planets because the ratio $s / s_{s}$ never drops below unity for $a_{1}<r<a_{2}$.
} 
term in the brackets on the right-hand side of equation (50) can be ignored. Most of the mutual inclination is due to the tilt of the smaller outer object, and the situation resembles that assumed in $\S 2$. Denoting the resonance position as $r=a_{2}(1+x)$, where $x \ll 1$, and approximating $b_{3 / 2}^{(1)} \approx$ $2 / \pi x^{2}$ gives

$$
x \approx\left(\frac{4}{21 \pi} \frac{\mu_{2}}{\alpha^{2} \mu_{1}}\right)^{1 / 3}=0.39\left(\frac{\mu_{2}}{\alpha^{2} \mu_{1}}\right)^{1 / 3}
$$

for the resonance location. The damping rate becomes

$$
\frac{1}{\sin I_{s}} \frac{d I_{s}}{d t} \approx-\frac{\pi}{8} \mu_{d} \mu_{2} \alpha_{2}^{3}\left[b_{3 / 2}^{(1)}\left(\alpha_{2}\right)\right]^{2}\left|\frac{\Omega}{r d s / d r}\right| \Omega_{2},
$$

which is identical to equation (17). If the gradient of $s(r)$ is due mostly to the $\mu_{2}$ term in equation (49), $|\Omega / r d s / d r| \approx$ $\pi x^{3} / \mu_{2}$ and equation (52) simplifies to

$$
\frac{1}{\sin I_{s}} \frac{d I_{s}}{d t} \approx-\frac{\mu_{d} \Omega_{2}}{2 x} \approx-1.3 \mu_{d} \Omega_{2}\left(\frac{\alpha \mu_{1}}{\mu_{2}}\right)^{1 / 3} .
$$

This rate is only weakly dependent on the planetary mass ratio. As an example, set $a_{1}=5 \mathrm{AU}, a_{2}=15 \mathrm{AU}$, and $\mu_{1}=10 \mu_{2}$, for which $x=0.26$. The resonance lies at $\sim 19$ $\mathrm{AU}$ and the characteristic damping timescale is $\tau_{\text {damp }} \approx 7 \mu_{d}^{-1}$ yr. A numerical evaluation of the Laplace coefficients in equation (48) puts the resonance closer to $21 \mathrm{AU}$ (Fig. 2).

Consider next the case where $\mu_{1}=\mu_{2}=\mu_{s}$, for which there is a nonnegligible inclination for both objects. To find the variation in their mutual inclination, $I_{s}$, the change in the $z$-component of the total angular momentum is used, that is, $\dot{L}_{1}+\dot{L}_{2}=|F|$. This is because no matter where the disk torque is applied, the mutual torque between the planets will redistribute the angular momentum so as to satisfy equation (45). The result can be written

$$
\begin{aligned}
\frac{1}{\sin I_{s}} \frac{d I_{s}}{d t}= & -\frac{\pi}{8} \mu_{d}\left(\frac{\mu_{1} \mu_{2}}{\mu_{1} \alpha^{1 / 4}+\mu_{2} \alpha^{-1 / 4}}\right) \\
& \times\left[\alpha_{1}^{1 / 2} \alpha_{2} b_{3 / 2}^{(1)}\left(\alpha_{2}\right)-\alpha_{2}^{1 / 2} \alpha_{1} b_{3 / 2}^{(1)}\left(\alpha_{1}\right)\right]^{2} \\
& \times\left|\frac{\Omega}{r d s / d r}\right| \sqrt{\Omega_{1} \Omega_{2}} .
\end{aligned}
$$

Because the outer planet has significant mass, we expect the distance to the external resonance to be somewhat comparable to the distance between the planets, so that $b_{3 / 2}^{(1)}\left(\alpha_{2}\right) \approx 3 \alpha_{2}, s \approx-\frac{3}{4} \mu_{s}\left(1+\alpha^{2}\right)\left(a_{2} / r\right)^{7 / 2} \Omega_{2}$, and

$$
\begin{gathered}
\frac{1}{\sin I_{s}} \frac{d I_{s}}{d t} \approx-\frac{9}{8} \pi \alpha^{1 / 2} f(\alpha) \mu_{d} \mu_{s}\left(\frac{a_{2}}{r}\right)^{5}\left|\frac{\Omega}{r d s / d r}\right| \Omega_{2}, \\
f(\alpha) \equiv\left(1-\alpha^{3 / 2}\right)^{2} /\left(1+\alpha^{1 / 2}\right) .
\end{gathered}
$$

The position of the resonance is $a_{2} / r=\alpha^{3 / 7}\left[\left(1+\alpha^{1 / 2}\right) /\right.$ $\left.\left(1+\alpha^{2}\right)\right]^{2 / 7} \equiv \alpha^{3 / 7} \tilde{f}(\alpha)$, while $r d s / d r \approx-7 s / 2$. Substituting for $s$ and $r$ gives

$$
\begin{gathered}
\frac{1}{\sin I_{s} \frac{d I_{s}}{d t}} \approx-\frac{3}{7} \pi \alpha^{25 / 14} g(\alpha) \mu_{d} \Omega_{2}, \\
g(\alpha) \equiv \frac{f \tilde{f}^{3}}{1+\alpha^{2}}=\frac{\left(1-\alpha^{3 / 2}\right)^{2}}{\left(1+\alpha^{2}\right)^{13 / 7}\left(1+\alpha^{1 / 2}\right)^{1 / 7}} .
\end{gathered}
$$

This implies a characteristic damping time of $\tau_{\text {damp }} \sim 0.12 g^{-1} \mu_{d}^{-1}\left(a_{2} / a_{1}\right)^{25 / 14} P_{2}$, where $P_{2}$ is the orbital period of the outer planet. Note that this does not depend on the planets' masses, because in equation (55) $\mu_{s}$ appears both in the nominator and in the denominator through $d s$ / $d r$. It is also interesting that both planets damp at the same rate, even though their individual torques and angular momenta are unequal. Again, this is due to communication between the planetary pair via their mutual torque (e.g., Agnor \& Ward 2002). Using the same semimajor axes as before, $g\left(\frac{1}{3}\right)=0.51,\left(\sin I_{s}\right)^{-1} d I_{s} / d t \approx 0.097 \mu_{d} \Omega_{2}$, and $\tau_{\text {damp }} \sim 140 \mu_{d}^{-1}$ yr. For this example, the external resonance lies at a distance $r_{r} \sim\left(a_{2} / a_{1}\right)^{3 / 7} a_{2} / \tilde{f}=1.6 a_{2}=24 \mathrm{AU}$, in pretty good agreement with the $\sim 25$ AU value of Figure 2 .

\subsection{Comparison with External VRs}

Finally, as mentioned at the outset, there is also an array of other $m>1$ VRs that simultaneously conspire to excite the secondary's inclination. The inclination excitation rate due to each $m$ th-order external resonance is given by BGT $84^{6}$ and can be written as

$$
\frac{1}{\sin I_{s}} \frac{d I_{s}}{d t} \approx \frac{3 m^{3}}{8 \pi} \mu_{s} \mu_{d}\left[K_{1}(4 / 3)\right]^{2} \Omega_{s},
$$

where $K_{1}$ is a modified Bessel function. Comparison with equation (17) reveals that equation (57) is smaller for $m \lesssim O\left(3(\Omega /|r d s / d r|)^{1 / 3}\right) \approx 3 \mu_{s}^{-1 / 3}$. The Jacobi constant is often invoked to argue that an accreting planetary embryo will tend to clear out a gap in a particle disk to a minimum size of $w_{\text {gap }} \gtrsim 2 \sqrt{3} R_{\mathrm{H}}$, where $R_{\mathrm{H}}=r\left(\mu_{s} / 3\right)^{1 / 3}$ is its Hill radius (e.g., Lissauer \& Stewart 1993). This places an upper limit on resonance order of $m_{\max } \sim 4 a / 3 w_{\text {gap }} \lesssim$ $0.5 \mu_{s}^{-1 / 3}$. The inordinate strength of the secular resonance can be traced to the long wavelength of apsidal waves, which allows their open spiral structure to couple well to the potential of the perturber over a large swath of the disk. Summing equation (57) over the $m \lesssim 4 a_{s} / 3 w$ resonances that lie beyond the gap width $w$ yields the cumulative inclination excitation rate,

$$
\frac{1}{\sin I_{s}} \frac{d I_{s}}{d t} \approx 0.012\left(\frac{a_{s}}{w}\right)^{4} \mu_{s} \mu_{d} \Omega_{s} .
$$

This implies a maximum excitation rate of $\left(\sin I_{s}\right)^{-1} d I_{S} /$ $d t \approx 3.6 \times 10^{-4} \mu_{d} \mu_{s}^{-1 / 3} \Omega_{s}$. If this rate is less than the rate of I-damping due to nodal wave generation (say, eq. [53] or [56]), secular resonant interaction with the disk will still stabilize the protoplanet's inclination and prevent it from growing. In the first case considered, both equation (53) and the maximum excitation rate have the same dependence on $\mu_{d} / \mu_{2}^{1 / 3}$, so that the ratio $\tau_{\text {damp }} / \tau_{\text {ex }} \approx 0.03\left[M_{1} /\left(1 M_{\oplus}\right)\right]^{-1 / 3}$. A more massive inner planet shortens the damping timescale by speeding up the precession rate, thereby causing the secular resonance to lie closer to the outer planet. For the second example above, $\tau_{\text {damp }} / \tau_{\text {ex }} \approx 0.3\left[M_{s} /\left(1 M_{\oplus}\right)\right]^{-1 / 3}$, and stability would be ensured for $M_{s} \gtrsim 2 \times 10^{-2} M_{\oplus}$, although lower masses could also be stable if they are able to open and maintain gaps larger than the minimum by shepherding action; this would depend on the specifics of

\footnotetext{
${ }^{6} \mathrm{We}$ assume the planets reside in gaps and so omit the portion of the BGT84 expression that is due to the satellite's recoil from the disk edge.
} 
the particle disk as well. Confining planetary embryos to low-inclination orbits may have important ramifications for their subsequent accretion rates.

\section{CONCLUSION}

An inclined precessing secondary will drive nodal waves in an adjacent disk at the site or sites of secular resonance. These are one-armed spiral bending waves that carry negative angular momentum; the reaction torque on the secondary damps its inclination. The wave pattern regresses at the rate of the secondary's node, and the slow pattern speed results in very little energy exchange with the disk and, consequently, only a minor effect on the semimajor axis of the secondary. The $(m=1)$ secular resonance is formally an inner vertical resonance associated with the slow pattern speed $(l=m-1)$ of equation (2). The $m>1$ versions of this resonance also damp $I$, but they fall (nearly) at the secondary's semimajor axis (Ward \& Hahn 1994). Thus, they are shut off if the object opens a gap in the disk. However, the secular resonance is sensitive to non-Keplerian components of the gravitational potential and can be displaced significantly from the secondary to lie in the disk outside a gap. The wavelengths of nodal waves are very long, and their open spiral structure couples efficiently to the forcing potential, resulting in an inordinately strong torque. Indeed, in some circumstances this single resonance can dominate the more numerous external vertical resonances and prevent them from exciting the secondary's inclination.

This work was supported by funds from NASA's Origins of Solar Systems and Planetary Geology and Geophysics Programs. We have benefited greatly from comments by Alar Toomre and an anonymous referee. This is contribution 1160 from the Lunar and Planetary Institute, which is operated by the Universities Space Research Association for NASA under cooperative agreement NCC 5-679.
Agnor, C. B., \& Ward, W. R. 2002, ApJ, 567, 579

Artymowicz, P. 1993, ApJ, 419, 166

1994, ApJ, 423, 581

Bertin, G., \& Mark, J. W.-K. 1980, A\&A, 88, 289

Borderies, N., Goldreich, P., \& Tremaine, S. 1984, ApJ, 284, 429 (BGT84)

Brouwer, D., \& Clemence, G. M. 1961, Methods of Celestial Mechanics (New York: Academic)

Goldreich, P., \& Sari, R. 2003, ApJ, 585, 1024

Goldreich, P., \& Tremaine, S. 1980, ApJ, 241, 425 (GT80)

Hunter, C., \& Toomre, A. 1969, ApJ, 155, 747

Lissauer, J. J., \& Stewart, G. R. 1993, in Protostars and Planets III, ed.

E. Levy \& J. I. Lunine (Tucson: Univ. Arizona Press), 1061

Rosen, P. A. 1989, Ph.D. thesis, Stanford Univ.

\section{REFERENCES}

Rosen, P. A., \& Lissauer, J. J. 1988, Science, 241, 690

Shu, F. H. 1984, in Planetary Rings, ed. R. Greenberg \& A. Brahic (Tucson: Univ. Arizona), 513

Shu, F. H., Cuzzi, J. N., \& Lissauer, J. J. 1983, Icarus, 53, 185

Tanaka, H., Takeuchi, T., \& Ward, W. R. 2002, ApJ, 565, 1257

Thorne, R. M. 1968, ApJ, 151, 671

Toomre, A. 1969, ApJ, 158, 899

Tremaine, S. 1998, AJ, 116, 2015

Ward, W. R. 1981, Icarus, 47, 234

.1988, Icarus, 73, 330

2003, ApJ, 584, L39

Ward, W. R., \& Hahn, J. M. 1994, Icarus, 110, 95 . 1998, AJ, 116, 489 